\title{
Protocol for a randomized controlled multicenter trial assessing the efficacy of leuprorelin for severe polycystic liver disease: the AGAINST-PLD study
}

\author{
S. E. Aapkes ${ }^{1}$, L. H. P. Bernts' ${ }^{2}$, A. P. van den Berg ${ }^{3}$, M. van den Berg ${ }^{4}$, H. Blokzijl ${ }^{3}$, A. E. P. Cantineau4 ${ }^{4}$, \\ M. D. A. van Gastel' ${ }^{1}$, R. J. de Haas ${ }^{5}$, P. Kappert ${ }^{5}$, R. U. Müller ${ }^{6}$, F. Nevens' ${ }^{7}$, R. Torra ${ }^{8}$, A. Visser ${ }^{9}$, J. P. H. Drenth ${ }^{2}$ and \\ R. T. Gansevoort ${ }^{1^{*}}$
}

\begin{abstract}
Background: In patients with severe polycystic liver disease (PLD), there is a need for new treatments. Estrogens and possibly other female sex hormones stimulate growth in PLD. In some patients, liver volume decreases after menopause. Female sex hormones could therefore be a target for therapy. The AGAINST-PLD study will examine the efficacy of the GnRH agonist leuprorelin, which blocks the production of estrogen and other sex hormones, to reduce liver growth in PLD.

Methods: The AGAINST-PLD study is an investigator-driven, multicenter, randomized controlled trial. Institutional review board (IRB) approval was received at the University Medical Center of Groningen and will be collected in other sites before opening these sites. Thirty-six female, pre-menopausal patients, with a very large liver volume for age (upper 10\% of the PLD population) and ongoing liver growth despite current treatment options will be randomized to direct start of leuprorelin or to 18 months standard of care and delayed start of leuprorelin. Leuprorelin is given as $3.75 \mathrm{mg}$ subcutaneously (s.c.) monthly for the first 3 months followed by 3-monthly depots of $11.25 \mathrm{mg}$ s.c. The trial duration is 36 months. MRI scans to measure liver volume will be performed at screening, 6 months, 18 months, 24 months and 36 months. In addition, blood will be drawn, DEXA-scans will be performed and questionnaires will be collected. This design enables comparison between patients on study treatment and standard of care (first 18 months) and within patients before and during treatment (whole trial). Main outcome is annualized liver growth rate compared between standard of care and study treatment. Secondary outcomes are PLD disease severity, change in liver growth within individuals and (serious) adverse events. The study is designed as a prospective open-label study with blinded endpoint assessment (PROBE).
\end{abstract}

Discussion: In this trial, we combined the expertise of hepatologist, nephrologists and gynecologists to study the effect of leuprorelin on liver growth in PLD. In this way, we hope to stop liver growth, reduce symptoms and reduce the need for liver transplantation in severe PLD.

\footnotetext{
*Correspondence: r.t.gansevoort@umcg.nl

${ }^{1}$ Department of Nephrology, University Medical Center Groningen,

University of Groningen, Groningen, The Netherlands

Full list of author information is available at the end of the article
} regulation or exceeds the permitted use, you will need to obtain permission directly from the copyright holder. To view a copy of this licence, visit http://creativecommons.org/licenses/by/4.0/. The Creative Commons Public Domain Dedication waiver (http://creativeco mmons.org/publicdomain/zero/1.0/) applies to the data made available in this article, unless otherwise stated in a credit line to the data. 
Trial registration Eudra CT number 2020-005949-16, registered at 15 Dec 2020. https://www.clinicaltrialsregister.eu/ctrsearch/search?query=2020-005949-16.

Keywords: Polycystic liver disease, GnRHa, Estrogen

\section{Background}

Polycystic liver disease (PLD) is a rare disease, characterized by the presence of $>10$ liver cysts [1]. Autosomal Dominant Polycystic Liver Disease (ADPLD) leads to PLD only and is caused by a mutation in PRKCSH, SEC63, LRP5, ALG8 or SEC61B [1]. Autosomal Dominant Polycystic Kidney Disease (ADPKD) can cause PLD but also leads to kidney cysts and renal function decline $[1-3]$ and is most frequently caused by a mutation in the $P K D 1$ or $P K D 2$ gene $[4,5]$. The PKD1 and PKD2 genes encode for ciliary proteins polycystin 1 and polycystin 2 , respectively, while the mutations in ADPLD affect maturation and trafficking of polycystin $1[3,4]$. The prevalence of ADPLD is $<1: 10.000$ [6], the point prevalence of clinically relevant ADPKD approximately 1:2500 [7], but not all ADPKD patients suffer from PLD [8].

PLD patients have a normal liver function in general, but large liver volumes can lead to serious physical and psychosocial complaints $[1,9]$. The large liver can compress the stomach and bowels, leading to early satiety, decreased food intake, weight loss, sarcopenia and constipation [1,9]. The increased intra-abdominal volume can lead to pain, and diaphragmatic, umbilical and inguinal herniation [1] and compression of vascular structures can lead to ascites, portal vein thrombosis or hepatovenous outflow obstruction [10]. The large, protruding abdomen may cause psychological problems, because of distorted body image and confronting questions about possible pregnancy $[1,9]$. This can result in decreased quality of life, and sometimes liver transplantation is the only and last resort treatment option $[11,12]$.

\section{An unmet need for new treatments}

Dependent on the cyst distribution, different treatments are possible. In case of several dominant liver cysts, aspiration or fenestration is used [1,9]. If liver cysts are clustered in a few liver segments, a hemihepatectomy can be performed [1]. In case of numerous liver cysts in all segments, surgical interventions are not possible and the mainstay of treatment is to halt natural growth [1, 9]. The only treatment now available to halt growth of liver cysts are somatostatin analogues, but in about $40 \%$ of patients treated with these drugs liver growth continues (Fig. 1) [13]. In these patients, the only treatment option that remains is a liver transplantation $[1,9,12]$. In the last 15 years, 1293 liver transplantations have been

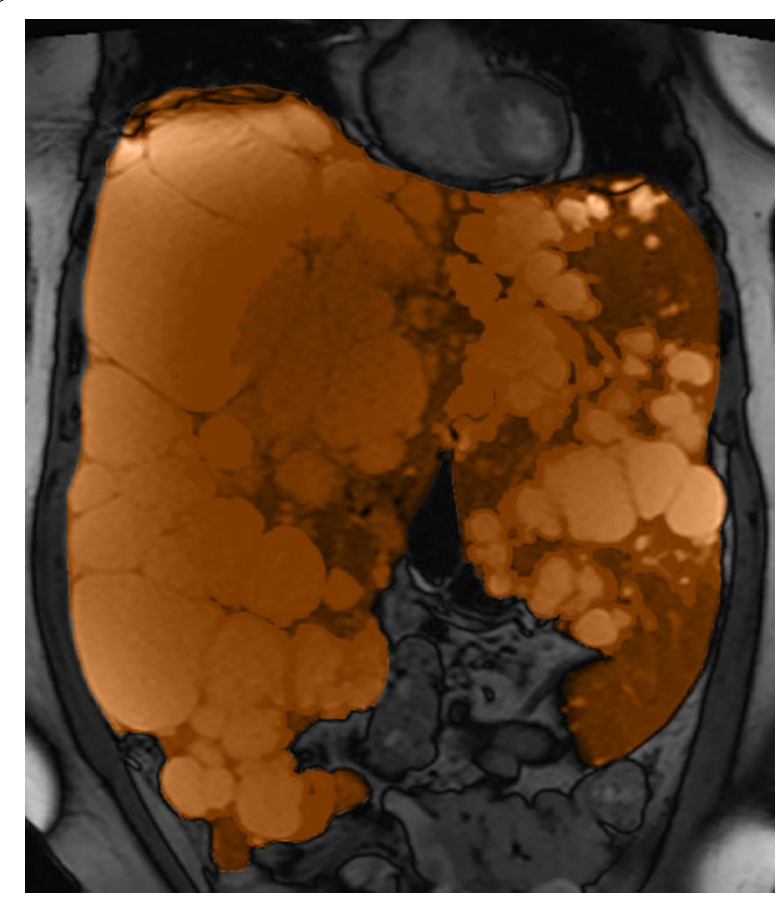

Fig. $1 \mathrm{MRI}$ scan (coronal T2-weighted image) of a patient with severe PLD and ongoing liver growth despite somatostatin analogue use, that would be eligible for the AGAINST-PLD study

performed in Europe to treat PLD [12]. Therefore, there is a continuing unmet need for new therapies in PLD.

\section{Female hormones in PLD}

Female sex is the most important risk factor for PLD [14]. About $90 \%$ of patients is female [14, 15]. Several factors indicate that female sex hormones stimulate cyst growth. First, estrogen supplementation leads to enhanced liver growth $[16,17]$ and after menopause, when estrogen and progesterone levels fall, liver volumes sometimes decrease spontaneously [18]. Second, estrogen receptors are present on hepatic cyst epithelium and in vitro, liver growth increases after estrogen supplementation and decreases after administration of estrogen blockers [19-22]. In a recent review paper, we explained in detail how the female sex hormones estrogen and progesterone may affect liver growth and how these hormones could be a target for therapies [22]. While there is a lot of evidence that estrogen affects liver cyst growth, it is difficult to disentangle the 
contributions of the individual sex hormones, like estrogen and progesterone, on PLD. Oral contraceptives mostly contain estrogen as well as progesterone and menopause also affects both hormones. Therefore, it is unclear whether other sex hormones than estrogen, such as progesterone, affect liver growth.

An ideal therapy should block all estrogen receptors and should block also other sex hormones, such as progesterone. For estrogen only, three different estrogen receptors [22] are present on hepatic cyst epithelium: ER- $\alpha$, ER- $\beta$ and the G-coupled protein estrogen receptor 1 (GPER-1) [19-23]. Selective estrogen receptor modulators (SERM), such as tamoxifen, act as agonist or antagonist on ER- $\alpha$ and $E R-\beta$, dependent on the surrounding tissue $[24,25]$. Currently it is not possible to predict the effects of SERMs in PLD. Besides, the effect on the GPER-1 receptor (inhibition or stimulation) is unclear. Therefore, in this trial, we aim to reduce liver growth in patients suffering from severe PLD using the Gonadotropin Releasing Hormone $(\mathrm{GnRH})$ agonist leuprorelin. This drug leads to cessation of the production of estrogen as well as progesterone and other female hormones, aiming to stop liver growth, but with medically induced menopause as logical adverse event [26].

\section{Objectives}

The main trial objective is:

- To determine whether lowering estrogen and progesterone levels with the GnRH agonist leuprorelin decreases liver growth rates $(\% / y)$ in pre-menopausal women with severe PLD when compared to similar women not receiving this treatment.

Secondary objectives are:

1. To assess the effect of leuprorelin on PLD disease severity, measured with the PLD-Questionnaire (PLD-Q)

2. To assess the change in liver growth within individuals before and during leuprorelin treatment

3. To assess the tolerability and incidence of (serious) adverse events.

Exploratory outcomes are:

1. To assess estrogen and progesterone levels

2. To assess differences in short term and long term effects of treatment

3. In case of ADPKD, to assess the effect on kidney growth and renal function decline.

\section{Methods and design}

Study design

The AGAINST-PLD study (A GnRH Agonist IN premenopausal women Study to Treat Polycystic Liver Disease) is a 3-year lasting, investigator driven, phase $2 \mathrm{~b}$ randomized controlled trial. Participants will be randomized between direct start or delayed start (18 months later) of leuprorelin treatment (Figs. 2, 3). Patients randomized to delayed start will receive standard of care in the first 18 months of the trial. Therefore, in the first 18 months of the trial, we can distinguish between the effect of treatment and the natural course of disease by comparing both treatment arms. Using data obtained during the second 18 months of the trial, when all patients are subjected to active treatment, we can compare liver growth rates within individuals before start of treatment and during treatment and obtain more information about safety and tolerability.

\section{Study setting}

The trial will be conducted according to the International Conference of Harmonization Good Clinical Practice Guidelines and adheres to the Declaration of Helsinki. IRB approval will be obtained in all participating centers. All participants must give their voluntary and written informed consent before any study related procedures could take place. Individuals who meet the entry criteria and complete the baseline visit will be enrolled in one of the five participating University Medical Centers: Groningen and Nijmegen, (the Netherlands), Cologne (Germany), Leuven (Belgium) and Barcelona (Spain).

\section{Patient selection}

Detailed inclusion criteria are given in Table 1, of which the most important are:

- Female patients, pre-menopausal

- Anti Mullerian Hormone (AMH) level>0.3 (a lower level indicates upcoming menopause)

- Age 18-45 years (inclusive)

- PLD with a very large liver for age, based on the upper $10 \%$ of volumes in our PLD registry $(n=1600)$ [27].

- One historical MRI or CT scan, performed 1-5 years before baseline, available to calculate liver growth before start of the study

- Ongoing liver growth, confirmed using a historical scan (a) obtained in routine clinical care and the scan at screening (b) with the liver volume in $\mathrm{ml}$ from scan $b-a>0$. 


\section{THE AGAINST-PLD STUDY}

$\underline{A} \underline{G}$ RH $\underline{A g o n i s t} \underline{\text { IN }}$ pre-menopausal women $\underline{\text { Study to }}$ Treat $\underline{P}$ olycystic Liver $\underline{\text { Disease }}$

Aiming to reduce liver volume and related complaints, increase quality of life and avoid liver transplantation in female patients suffering from severe PLD

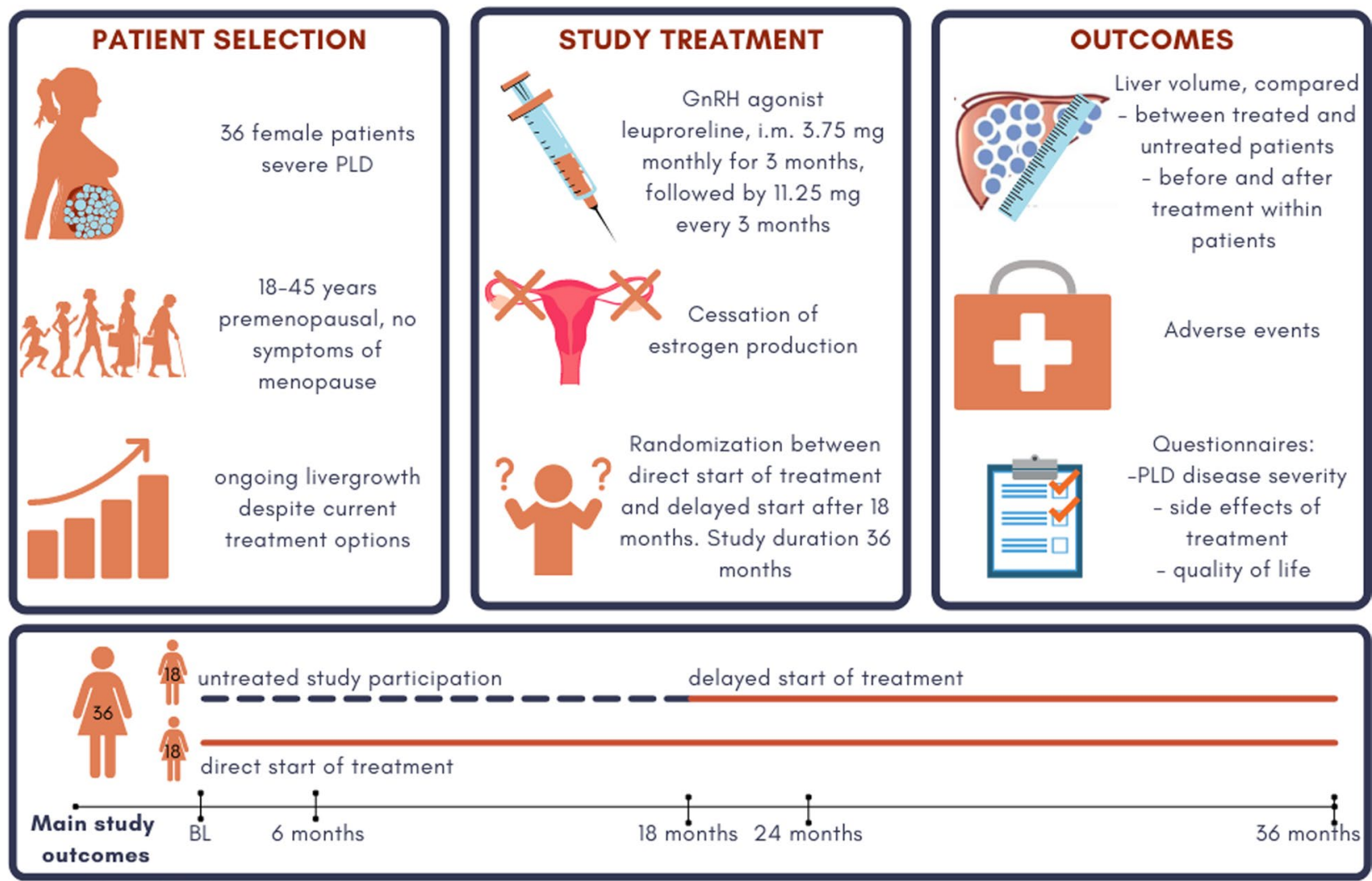

Fig. 2 Infographic about the AGAINST-PLD study

- Use of a somatostatin analogue (only proven effective therapy currently available) or reason not to use such drug (e.g. not tolerated, ineffective, not available)

Most important exclusion criteria are:

- Symptoms of (upcoming) menopause, e.g. hot flushes

- Active desire to have children during study period

- Use of exogenous estrogen and/or progesterone

- Contra-indication for leuprorelin

\section{Randomization}

Participants will be randomized 1:1 between direct and delayed start (after 18 months) of treatment using an online Interactive Response System (IXRS) tool, stratified for somatostatin analogue use (yes/no) and age $(<40$ and $\geq 40$ years).

\section{Intervention}

Leuprorelin is a synthetical analogue of $\mathrm{GnRH}$ and strongly stimulates the pituitary resulting in desensitization. Thus, the production of luteinizing hormone $(\mathrm{LH})$ and follicle stimulating hormone (FSH) by the pituitary stops, which leads to cessation of the production of estrogen and progesterone by the ovaries.

Leuprorelin will be started using monthly injections of $3.75 \mathrm{mg}$, and if tolerated after 3 months, treatment regimen will be switched to 3-monthly injections of $11.25 \mathrm{mg}$. Depending on patients' preferences, participants will be trained to self-administer the injections at home or injections will be administered by study personnel. 


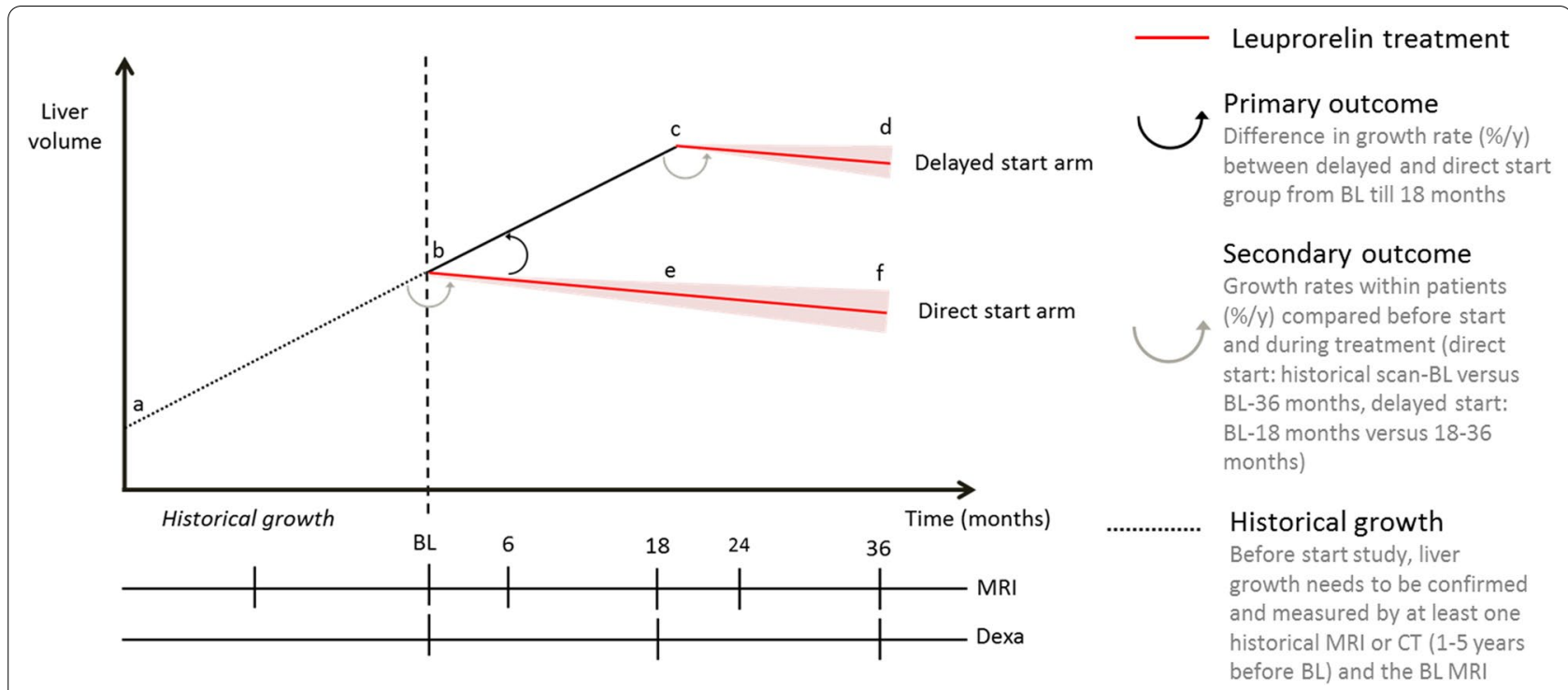

Fig. 3 Study design of the AGAINST-PLD study. Patients are randomized between direct and delayed start (after 18 months) of treatment. a Historical scan, $\mathbf{b}$ baseline scan, $\mathbf{a}, \mathbf{b}$ historical growth. Primairy outcome, comparison between growth on treatment and without treatment in the first 18 months is $\mathbf{b}$, c versus $\mathbf{b}$, e. Secondary outcome, growth within individuals before and during treatment is $\mathbf{b}, \mathbf{c}$ versus $\mathbf{c}, \mathbf{d}$ and $\mathbf{a}, \mathbf{b}$ versus $\mathbf{b}, \mathbf{e}$

\section{Concomitant medication}

For massive PLD, somatostatin analogues are currently the only evidence-based treatment. Therefore, somatostatin analogues should be used, tried, or have been considered before inclusion in the AGAINST-PLD trial. Patients are thus allowed to use a somatostatin analogue during the trial (see inclusion criteria, Table 1). During the study, start or stop of somatostatin analogues as well as surgical procedures to reduce liver volume should be avoided unless there are important reasons to do so, because this could interfere with the assessment of the primary outcome, liver growth.

Use of estrogen- and or progesterone containing medication is not allowed during the trial period, because it could interfere with the effect of the study treatment. Blood pressure treatment is targeted below $130 / 80 \mathrm{mmHg}$ with RAAS-inhibitors as the firstchoice agents. Calcium vitamin D (500 mg/800 IE) will be prescribed to all patients in the study to prevent osteoporosis.

\section{Outcome parameters \\ Primary outcome}

- Liver growth $(\% / y)$, calculated over the first 18 months of the trial and compared between the direct start group (on treatment) and delayed start group (on standard of care), b-e versus b-c, Fig. 3.

\section{Secondary outcomes}

1. Change in PLD disease severity, assessed by the validated PLD-Q questionnaire, calculated over the first 18 months of the trial and compared between the direct start group (on treatment) and delayed start group (on standard of care).

2. Liver growth rates within individuals compared before treatment and during treatment. In the direct start group, historical growth rates (Fig. 3a, b) will be compared with growth rate during 18 months of treatment (b-e) and in the delayed start group, growth rates during the first 18 months of the trial (untreated, b-c) will be compared with the last 18 months of the trial (treated, $\mathrm{c}-\mathrm{d}$ ).

3. The incidence of (serious) adverse events, compared between the direct and delayed start group in the first 18 months of treatment.

\section{Exploratory outcomes}

- Height adjusted liver volumes, compared between the direct and delayed start group after 18 months

- Estradiol, progesterone, AMH, LH and FSH levels at baseline, 6, 18, 24 and 36 months 
Table 1 Overview of in- and exclusion criteria for the AGAINST-PLD study

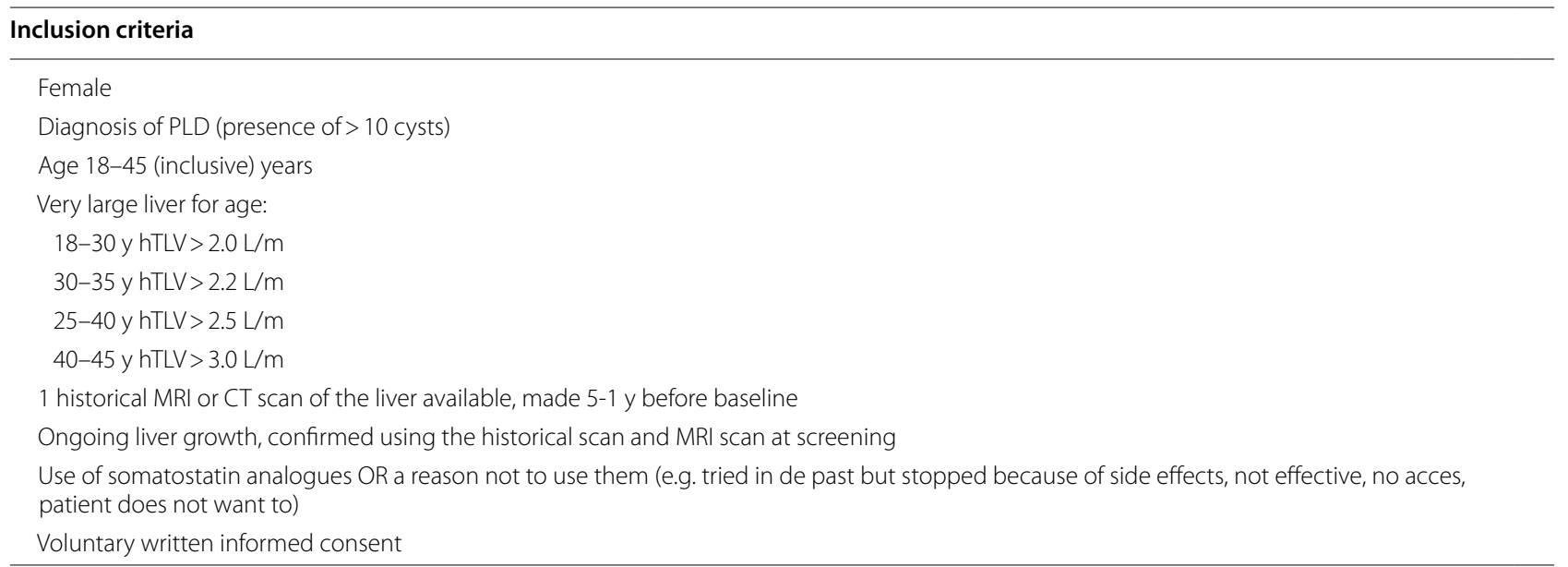

\section{Exclusion criteria}

Post menopausal status or (vasomotor) symptoms indicating upcoming menopause

$\mathrm{AMH}<0.03 \mathrm{ng} / \mathrm{ml}$ at screening

Active desire to have children, pregnancy or breast-feeding

Contra-indications for leuprorelin, such as cardiovascular disease or osteoporosis

Liver transplantation expected within 1.5 years

Use of oral estrogen or progesterone containing medication

Contra-indications for MRI

Chronic use of immunosupressive agents

Severe hypertension (systolic pressure $\geq 160$ and/or diastolic pressure $\geq 100 \mathrm{mmHg}$ )

Clinically significant uncontrolled medical condition that, in the opinion of the investigator, would put the safety of the patient at risk though participation, or which would affect efficacy or safety analysis, such as, but not limited to, recurrent cholangitis, recurrent ascites, hepato-venous outflow obstruction, (history of) depression

Participation in other interventional studies at the same time

\section{Exclusion criteria related to the historical MRI/CT scan}

Start or stop of liver volume reducing therapy (medication e.g. somatostain analogues or surgical interventions) between the historical scan and screening

Historical scan is performed $<3$ months after start or stop of volume reducing therapy (either a somatostatin analogue or surgical treatments)

- Difference between acute and chronic effects of treatment on liver growth as assessed by a mixed models analysis comparing liver growth rate in the first 6 months of treatment compared to the next 12 months of treatment.

\section{With respect to tolerability:}

- Quality of life, using the validated 36-Item Short Form Health Survey (SF-36) and the Beck Depression Inventory II (BD-II) questionnaires

- Menopause related complaints: using the Menopause Specific Quality of Life (MENQOL) questionnaire
- Bone density: T-score on Dual Energy X-ray Absorptiometry (DEXA) scans will be compared between direct start group and delayed start group after 18 months.

For patients with polycystic kidney disease (PKD) also:

- Kidney growth $(\% / y)$ calculated over the first 18 months of the trial compared between direct and delayed start group

- Kidney growth rates within individuals compared before treatment and during treatment

- Differences between acute and chronic effects of treatment on kidney growth 
- Renal function decline before and during treatment.

\section{Sample size calculation}

In our own DIPAK 1 trial [13], the mean liver growth rate in patients meeting the inclusion criteria was $+5.8 \% \pm 2.8 \%$ per 18 months. Since the information on liver growth rate in this specific group is scarce, we conservatively doubled the SD to ensure enough power. We aim at fully stopping liver growth. Using $\beta=80 \%$, $\alpha=0.05$, and incorporating a $20 \%$ drop-out rate, inclusion of 36 patients is needed to show this effect. In other proof-of-concept studies in PLD, similar numbers of patients were included $[28,29]$.

\section{Recruitment}

Patients will be recruited from outpatient clinics of the participating centers or referred by other centers. All participating centers are expertise centers on polycystic liver and/or polycystic kidney disease and see many patients that meet the inclusion criteria for the trial. We planned 24 months for the recruitment period.

\section{Data collection and management}

The study consists of one or two screening visits, nine hospital visits and one phone call. During short visits (3, 12, 15, 21 months), a small set of local laboratory measurements, vital signs, AEs and concomitant medication will be assessed. At larger visits (BL, 6, 18, 24 and 36 months) in addition MRI scans, extensive lab and questionnaires will be performed. DEXA scans are made at baseline, halfway and end of the study (Figs. 3, 4). Data will be entered in a web-based electronic case report form to ensure correct and timely data collection in a central database.

\section{MRI scans}

Liver and kidney volumes (the latter in ADPKD patients only) will be measured using an MRI scan at screening, 6 , 18,24 and 36 months. MRI scans will be performed using a standardized protocol, including a coronal T2-weighted single-shot fast gradient spin-echo sequence with fatsaturation with a slice thickness of $3 \mathrm{~mm}$ which is the primary sequence to determine liver volumes. In addition, a transversal T2-weighted single shot fast gradient spin-echo sequence with fat suppression and a coronal T1-DIXON-VIBE spoiled gradient echo sequence will be obtained. After pseudonymization, MRI scans will be sent to the central reading facility, where images will

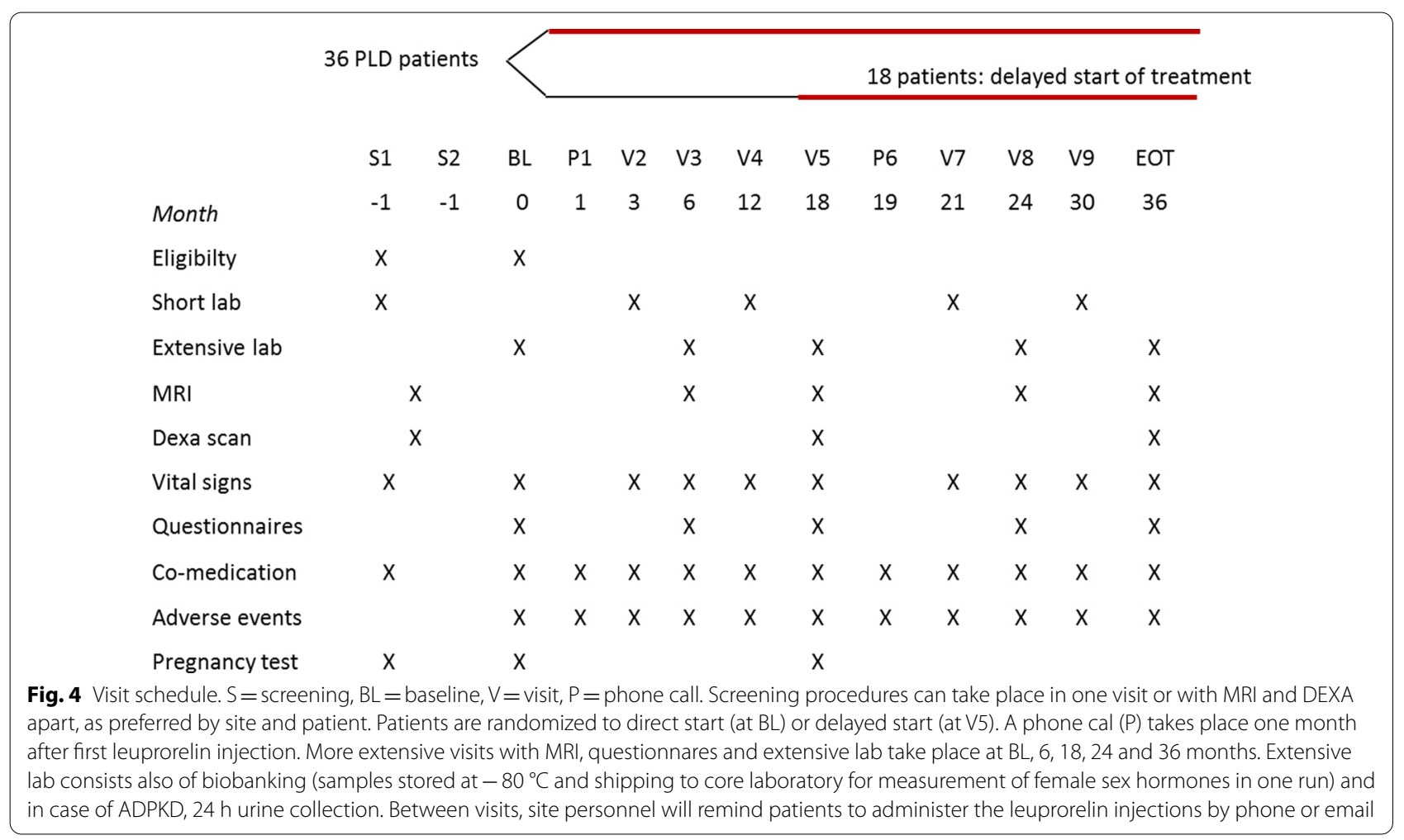


be analyzed blinded for patient, time and treatment by trained observers using an automated MRI program [30]. To ensure that valid MR images are obtained, quality control will be performed within $72 \mathrm{~h}$ by trained personnel. In case a scan is rejected, it will be repeated.

\section{Laboratory measurements}

For a detailed overview of collected samples, see Fig. 4. At all visits, a limited set of laboratory measurements will be performed. At baseline and the visits at month 6 , 18,24 and 36, more extensive laboratory measurements, biobanking and in ADPKD patients, 24-h urine samples will be collected. Except form biobanking samples, laboratory samples will be analyzed locally. Biobanking samples will be stored at $-80^{\circ} \mathrm{C}$ and shipped to the core laboratory at the end of the trial to measure estrogen, progesterone, $\mathrm{AMH}, \mathrm{FSH}$ and $\mathrm{LH}$ in one run, to minimize interlaboratory and interassay variation.

\section{Vital signs}

During every visit we will measure blood pressure, weight and abdominal circumference. Height will be collected at baseline. Upper arm circumference of the non-dominant arm will be measured every visit because this is one of the exception criteria for the MELD score [31].

\section{Questionnaires}

Questionnaires will be sent digitally to the patient at screening, 6, 18, 24 and 36 months. Quality of life will be assessed using the SF-36 and BD-II questionnaires, PLD disease severity using the validated PLD-Q questionnaire and menopause related complaints using the MENQOL questionnaire [32, 33].

\section{Criteria for withdrawal}

Subjects can leave the study at any time for any reason if they wish to do so without any consequences. Patients that drop-out from the trial within the first 3 months from baseline will be replaced and not be part of the intention to treat analysis. Patients that drop-out from the trial later on will not be replaced by new patients and will be asked for an early-end of treatment. The investigator can withdraw a subject from the study for urgent medical reasons, such as (but not limited to): occurrence of a concomitant disease in which leuprorelin is contra-indicated, pregnancy, need of treatment that is contra-indicated during the trial (such as estrogens), lostto-follow up or, by the opinion of the treating physician, high need for surgical volume reducing treatments. If a patient that is registered on the waiting list receives a transplant offer, this patient will also be withdrawn from the trial.

\section{Statistics \\ Statistical analysis}

Analyses are performed after completion of the study. For the primary outcome, liver growth during the first 18 months of the trial compared between direct and delayed starters, an unpaired t-test will be used. In case of missing data, last information will be used adjusted for the duration of follow up. For questionnaires, the delta in scores during the first 18 months of the trial will be compared between direct and delayed starters. To compare liver growth within individuals before and during treatment, a paired t-test will be used. Adverse events will be categorized and the number of adverse events per category will be compared between direct and delayed starters using an unpaired $t$-test.

For all primary and secondary analyses, we will perform pre-defined sensitivity analysis to adjust for age, disease type (ADPKD vs. ADPLD), total liver volume at baseline, total kidney volume at baseline, use of somatostatin analogues, and test differences in treatment effects among these variables (interaction). In a sensitivity analysis we will test for differences in the outcome variables between the first 18 months of the trial and the second 18 months of the trial in the direct start group, using a breakpoint analysis. All analyses will be performed based on an intention to treat principle. As a sensitivity analysis, we will perform a per-protocol analysis.

In an additional analysis we will explore whether liver growth is linear and whether the liver growth rate during treatment is different in the first 6 months of treatment compared to the 12 months thereafter, using a multiple mixed model.

\section{Discussion}

The AGAINST-PLD study will assess the effect of the $\mathrm{GnRH}$-agonist leuprorelin on liver growth in pre-menopausal patients with severe PLD. If effective, leuprorelin may help to stop disease progression in female PLD patients at risk of rapid disease progression. Ultimately, this may avoid liver transplantations, which is desirable in view of the donor shortage and the procedure related morbidity and mortality [12].

We study the effect of anti-estrogenic therapy in PLD, a novel and promising target, using a broad proof-of-concept approach. To reiterate a recent literature review [22], estrogens have a cyst growth promoting effect and the idea to study estrogen inhibition in female PLD patients has been coined several times [3, 20,22,34]. To inhibit the effect of estrogens, several approaches could be used [22]. We chose for a GnRH-agonist, because this is the 
only way currently available to inhibit all three types of estrogen receptors and also block the effects of other female hormones such as progesterone. For other treatments, such as tamoxifen (a selective estrogen receptor modulator) it is so far unclear whether they would inhibit, stimulate or either have no effect on estrogen receptors in PLD and they would not block other female hormones which possibly also affect liver growth [22].

Since the study treatment ceases the menstruation and can lead to menopause-related complaints, blinding is not possible and as a consequence we adopted a prospective open-label with blinded endpoint assessment (PROBE) design. Randomization between direct and delayed start of treatment makes it possible to compare treated and untreated patients, but also to compare liver growth before and during treatment within patients, and it will render additional safety and tolerability data. Finally, the design meets the urgency felt by patients, stating that they would not participate if they could be in a control group without treatment.

\section{Efficacy-safety ratio}

Leuprorelin blocks the production of all female sex hormones and can have several side effects that we know from menopause, such as hot flushes, night sweats, mood swings, vaginal dryness and disturbed sleep [26]. On the long term, estrogen depletion could increase the risk of osteoporosis and cardiovascular events [35, 36].

We tried to enhance the tolerability and the efficacysafety ratio of the study medication in several ways. First, only patients with the highest need for new treatment options are included in this study. The target population consist of patients who, despite current treatment options, will mostly need a liver transplantation in the future if the course of the disease cannot be altered. We discussed the design of this trial in a focus group with eight patients meeting the inclusion criteria and with de Dutch Liver Patient Foundation. They felt that the burden of the disease is high and the perspective grim, and most patients thought that the potential of efficacy of a new treatment outweighs side effects.

Second, with the help of patient focus groups we adjusted the study design to the preferences and needs of patients to be included, especially to limit adverse events related to the mechanism of action of GnRH agonists. A potential option to reduce side effects would be to add a low-dose estrogen and/or progesterone as "addback therapy", as is used in GnRH agonist treatment for endometriosis [26]. However, because this is a proof-ofconcept trial testing whether estrogen depletion curtails liver growth, we decided to not allow add-back therapy. Instead, we added that non-hormonal treatments can be used to treat side effects, such as clonidine in case of hot flushes and venlafaxine in case of mood swings. In addition, also at the request of the patients in the focus group, a gynecologist will be available at all study sites to counsel patients before and during the study, at some sites supported by a trained nurse as "menopause coach".

On the longer term, estrogen depletion could lead to an increased risk of osteoporosis and a slightly enhanced cardiovascular risk $[26,35,36]$. With a treatment duration of 18 or 36 months, we do not expect large detrimental effects on the long term. However, to ensure optimal safety, patients with a history of cardiovascular disease and a low bone-density at screening will be excluded from the trial and during the trial blood pressure, cholesterol levels and bone-density will be monitored (Fig. 4). In addition, all patients will receive advise on healthy food intake and calcium and vitamin D supplementation.

\section{Interim analysis}

To safeguard a favorable efficacy-safety ratio during the trial, a Data Safety and Monitoring Board will meet regularly to discuss the progress and interim results. A formal interim analysis for safety and futility will be performed when $50 \%$ of the patients $(n=18)$ have reached 18 months, the time point at which the primary outcome is assessed.

\section{Perspective}

In case the trial is positive, leuprorelin treatment will at first be especially indicated in patients suffering from severe, symptomatic PLD given the nature of the intervention and the expected side-effects. However, when more experience is gained, and leuprorelin also shows a good efficacy-safety ratio in a real life setting, the use of this drug could be extended to patients with less severe forms of PLD, that notwithstanding are symptomatic.

With the AGAINST-PLD study, we venture outside the somatostatin analogue era and explore a promising new target for intervention to stop PLD progression: inhibition of female sex hormones. In this trial we will include patients with the highest need for treatment. In this way we hope to be able to reduce liver growth, and associated symptoms, an urgent unmet need in PLD.

\footnotetext{
Abbreviations

ADPKD: Autosomal Dominant Polycystic Kidney Disease; ADPLD: Autosomal Dominant Polycystic Liver Disease; AMH: Anti Mullerian Hormone; BD-II: Beck Depression Inventory Questionnaire; CT: Computed tomography; DEXA: Dual Energy X-ray Absorptiometry; DIPAK: Developing Intervention strategies to halt Progression of Autosomal Dominant Polycystic Kidney Disease; ER: Estrogen receptor; FSH: Follicle stimulating hormone; GnRH: Gonadotropin receptor hormone; GPER: G-coupled protein estrogen receptor; LH: Luteinizing hormone; MENQOL: Menopause Specific Quality of Life; MRI: Magnetic resonance imaging; PLD: Polycystic liver disease; PLD-Q: Polycystic Liver
} 
Disease Questionnaire; PROBE: Prospective open label study with blinded endpoint assessment.

\section{Acknowledgements}

We thank J. Willemse, director of the Dutch Liver Patient Foundation, for her commitment in the preparations of this trial.

\section{Authors' contributions}

Study design and protocol development: SEA, LHPB, JPHD and RTG. Gynecological aspects of study design: MvdB and AEPC. Imaging aspects of study design: PK, RJdH and MDAvG. Patient involvement: AV. Local investigators participating in the design and conduct of the trial: $A P v d B, H B, R U M, F N$ and RT. All authors read and approved the final manuscript.

\section{Funding}

The AGAINST-PLD study is funded by unrestricted grants from the Dutch Government (ZonMW grant 10140261910001) and Abbvie (SA-003047 (ACANETH-20-01)) (providing study medication free of charge). Neither organization had any role in the conception of this study, nor in the writing of the study protocol and this manuscript.

\section{Availability of data and materials}

De-identified individual participant data collected during the trial will be made available upon request to researchers who provide a methodologically sound proposal and whose use of the data has been approved by the AGAINST-PLD Steering Committee.

\section{Declarations}

\section{Ethics approval and consent to participate}

Ethics approval was obtained before start of the trial by the Internal Review Board (IRB) from the University Medical Center of Groningen, METC number METc 2021/119. All participants must give their voluntary and written informed consent before any study related procedures could take place.

\section{Consent for publication}

Not applicable.

\section{Competing interests}

Dr. Gansevoort received grant support and fees for serving on advisory boards and steering committees from Galapagos, IPSEN, Otsuka Pharmaceuticals and Sanofi-Genzyme. In addition, Dr. Gansevoort holds the Orphan Medicinal Product Designation status at the European Medicines Agency for lanreotide as treatment for kidney function decline in ADPKD (EMA/OD/027/15). Dr. Drenth has received grant support and fees for serving on advisory boards and consultancy from IPSEN and Novartis. All money is paid to their institutions. No other potential conflict of interest relevant to this article was reported. The Dept. II of Internal Medicine (University of Cologne) received research funding from Otsuka Pharmaceuticals and ThermoFisherScientific

\section{Author details}

${ }^{1}$ Department of Nephrology, University Medical Center Groningen, University of Groningen, Groningen, The Netherlands. ${ }^{2}$ Department of Gastroenterology and Hepatology, Radboud University Medical Center, Nijmegen, The Netherlands. ${ }^{3}$ Department of Gastroenterology and Hepatology, University Medical Center Groningen, University of Groningen, Groningen, The Netherlands. ${ }^{4}$ Department of Obstetrics and Gynecology, University Medical Center Groningen, University of Groningen, Groningen, The Netherlands. ${ }^{5}$ Department of Radiology, University Medical Center Groningen, University of Groningen, Groningen, The Netherlands. ${ }^{6}$ Department II of Internal Medicine and Center for Molecular Medicine Cologne, Faculty of Medicine, University Hospital Cologne, University of Cologne, Kerpener Straße 62, 50937 Cologne, Germany. ${ }^{7}$ Department of Gastroenterology and Hepatology, Universiteitsziekenhuis Leuven, Leuven, Belgium. ${ }^{8}$ Department of Nephrology, Fundacio Puigvert, Barcelona, Spain. ${ }^{9}$ Department of Applied Health Sciences, University Medical Center Groningen, University of Groningen, Groningen, The Netherlands.

Received: 31 January 2022 Accepted: 6 February 2022

Published online: 25 February 2022

\section{References}

1. Van Aerts RMM, Van De Laarschot LFM, Banales JM, Drenth JPH. Clinical management of polycystic liver disease. J Hepatol. 2018;68:827-37.

2. Bae KT, Zhu F, Chapman AB, Torres VE, Grantham JJ, Guay-Woodford LM, et al. Magnetic resonance imaging evaluation of hepatic cysts in early autosomal-dominant polycystic kidney disease: the Consortium for Radiologic Imaging Studies of Polycystic Kidney Disease cohort. Clin J Am Soc Nephrol. 2006;1:64-9.

3. Barten TRM, Bernts LHP, Drenth JPH, Gevers TJG. New insights into targeting hepatic cystogenesis in autosomal dominant polycystic liver and kidney disease. Expert Opin Ther Targets. 2020;24:589-99.

4. Harris PC, Torres VE, Harris PC, Torres VE. Genetic mechanisms and signaling pathways in autosomal dominant polycystic kidney disease. J Clin Investig. 2014;124:2315-24.

5. Cornec-Le Gall E, Torres VE, Harris PC. Genetic complexity of autosomal dominant polycystic kidney and liver diseases. J Am Soc Nephrol. 2018;29:13-23.

6. Suwabe T, Chamberlain AM, Killian JM, King BF, Gregory AV, Madsen CD, et al. Epidemiology of autosomal-dominant polycystic liver disease in Olmsted county. JHEP Rep. 2020;2:100166.

7. Willey CJ, Blais JD, Hall AK, Krasa HB, Makin AJ, Czerwiec FS. Prevalence of autosomal dominant polycystic kidney disease in the European Union. Nephrol Dial Transplant. 2017;32:1356-63.

8. Meijer E, Visser FW, Van Aerts RMM, Blijdorp CJ, Casteleijn NF, D'Agnolo HMA, et al. Effect of lanreotide on kidney function in patients with autosomal dominant polycystic kidney disease the DIPAK 1 randomized clinical trial. JAMA. 2018;320:2010-9.

9. Cnossen WR, Drenth JPH. Polycystic liver disease: an overview of pathogenesis, clinical manifestations and management. Orphanet J Rare Dis. 2014;9:69.

10. Bernts LHP, Drenth JPH, Tjwa ETTL. Management of portal hypertension and ascites in polycystic liver disease. Liver Int. 2019;39:2024-33.

11. Neijenhuis MK, Kievit W, Verheesen SMH, D'Agnolo HM, Gevers TJG, Drenth JPH. Impact of liver volume on polycystic liver disease-related symptoms and quality of life. United Eur Gastroenterol J. 2018;6:81-8.

12. Adam R, Karam V, Cailliez V, O Grady JG, Mirza D, Cherqui D, et al. 2018 annual report of the European Liver Transplant Registry (ELTR) - 50-year evolution of liver transplantation. Transpl Int. 2018;31:1293-317.

13. van Aerts RMM, Kievit W, D'Agnolo HMA, Blijdorp CJ, Casteleijn NF, Dekker SEl, et al. Lanreotide reduces liver growth in patients with autosomal dominant polycystic liver and kidney disease. Gastroenterology. 2019;157:481-91.

14. van Aerts RMM, Kievit W, de Jong ME, Ahn C, Bañales JM, Reiterová J, et al. Severity in polycystic liver disease is associated with aetiology and female gender: results of the International PLD Registry. Liver Int. 2019;39:575-82.

15. Van Keimpema L, De Koning DB, Van Hoek B, Van Den Berg AP, Van Oijen $\mathrm{MGH}$, De Man RA, et al. Patients with isolated polycystic liver disease referred to liver centres: clinical characterization of 137 cases. Liver Int. 2011;31:92-8

16. Sherstha R, McKinley C, Russ P, Scherzinger A, Bronner T, Showalter R, et al Postmenopausal estrogen therapy selectively stimulates hepatic enlargement in women with autosomal dominant polycystic kidney disease. Hepatology. 1997;26:1282-6.

17. van Aerts RMM, Bernts LHP, Gevers TJG, Kievit W, Koopmans L, Nieboer TE, et al. Estrogen-containing oral contraceptives are associated with polycystic liver disease severity in premenopausal patients. Clin Pharmacol Ther. 2019;106:1338-45.

18. Chebib FT, Jung Y, Heyer CM, Irazabal MV, Hogan MC, Harris PC, et al. Effect of genotype on the severity and volume progression of polycystic liver disease in autosomal dominant polycystic kidney disease. Nephrol Dial Transplant. 2016;31:952-60.

19. Alvaro D, Alpini G, Onori P, Franchitto A, Glaser SS, Le Sage G, et al. Alfa and beta estrogen receptors and the biliary tree. Mol Cell Endocrinol. 2002;193:105-8.

20. Alvaro D, Mancino MG, Onori P, Franchitto A, Alpini G, Francis $H$, et al. Estrogens and the pathophysiology of the biliary tree. World J Gastroenterol. 2006;12:3537-45.

21. Alvaro D, Onori P, Alpini G, Franchitto A, Jefferson DM, Torrice A, et al. Morphological and functional features of hepatic cyst epithelium in autosomal dominant polycystic kidney disease. Am J Pathol. 2008;172:321-32. 
22. Aapkes SE, Bernts LHP, Barten TRM, van den Berg M, Gansevoort RT, Drenth JPH. Estrogens in polycystic liver disease: a target for future therapies? Liver Int. 2021;41:2009-19.

23. Onori P, Franchitto A, Mancinelli R, Carpino G, Alvaro D, Francis $H$, et al. Polycystic liver diseases. Dig Liver Dis. 2010;42:261-71.

24. Pinkerton JV, Conner EA. Beyond estrogen: advances in tissue selective estrogen complexes and selective estrogen receptor modulators. Climacteric. 2019;22:140-7.

25. Ellis AJ, Hendrick VM, Williams R, Komm BS. Selective estrogen receptor modulators in clinical practice: a safety overview. Expert Opin Drug Saf. 2015;14:921-34

26. Tarlatzis $\mathrm{BC}$, Bili H. Safety of $\mathrm{GnRH}$ agonists and antagonist. Expert Opin Drug Saf. 2004;3:39-46.

27. D'Agnolo HMA, Kievit W, Andrade RJ, Karlsen TH, Wedemeyer H, Drenth JPH. Creating an effective clinical registry for rare diseases. United Eur Gastroenterol J. 2016:4:333-8.

28. Gevers TJG, Inthout J, Caroli A, Ruggenenti P, Hogan MC, Torres VE, et al. Young women with polycystic liver disease respond best to somatostatin analogues: a pooled analysis of individual patient data. Gastroenterology. 2013;145:357-65.

29. Hogan MC, Masyuk TV, Page LJ, Kubly VJ, Bergstralh EJ, Li X, et al. Randomized clinical trial of long-acting somatostatin for autosomal dominant polycystic kidney and liver disease. J Am Soc Nephrol. 2010;21:1052-61.

30. van Gastel MDA, Edwards ME, Torres VE, Erickson BJ, Gansevoort RT, Kline TL. Automatic measurement of kidney and liver volumes from MR images of patients affected by autosomal dominant polycystic kidney disease. J Am Soc Nephrol. 2019:30:1514-22.

31. Eurotransplant. Chapter 5-ET Liver Allocation System (ELAS). Eurotransplant man. 2019;103-4. Available from https://www.eurotransplant.org/ cms/index.php?page=et_manual.

32. Neijenhuis MK, Gevers TJG, Hogan MC, Kamath PS, Wijnands TFM, van den Ouweland RCPM, et al. Development and validation of a diseasespecific questionnaire to assess patient-reported symptoms in polycystic liver disease. Hepatology. 2016;64:151-60.

33. Sydora BC, Fast H, Campbell S, Yuksel N, Lewis JE, Ross S. Use of the Menopause-Specific Quality of Life (MENQOL) questionnaire in research and clinical practice: a comprehensive scoping review. Menopause. 2016;23:1038-51.

34. Perugorria MJ, MasyukTV, Marin JJ, Marzioni M, Bujanda L, Larusso NF, et al. Polycystic liver diseases: advanced insights into the molecular mechanisms. Nat Rev Gastroenterol Hepatol. 2014;11:750-61.

35. Muka T, Oliver-Williams C, Kunutsor S, Laven JSE, Fauser BCJM, Chowdhury $\mathrm{R}$, et al. Association of age at onset of menopause and time since onset of menopause with cardiovascular outcomes, intermediate vascular traits, and all-cause mortality: a systematic review and meta-analysis. JAMA Cardiol. 2016;1:767-76.

36. Makita K, Ishitani K, Ohta H, Horiguchi F, Nozawa S. Long-term effects on bone mineral density and bone metabolism of 6 months' treatment with gonadotropin-releasing hormone analogues in Japanese women: comparison of buserelin acetate with leuprolide acetate. J Bone Miner Metab. 2005;23:389-94.

\section{Publisher's Note}

Springer Nature remains neutral with regard to jurisdictional claims in published maps and institutional affiliations.

Ready to submit your research? Choose BMC and benefit from:

- fast, convenient online submission

- thorough peer review by experienced researchers in your field

- rapid publication on acceptance

- support for research data, including large and complex data types

- gold Open Access which fosters wider collaboration and increased citations

- maximum visibility for your research: over 100M website views per year

At BMC, research is always in progress.

Learn more biomedcentral.com/submissions 